Bangladesh J. Plant Taxon. 27(1): 137-152, 2020 (June)

(C) 2020 Bangladesh Association of Plant Taxonomists

\title{
A SURVEY ON THE FLORAL DIVERSITY OF RURAL AREAS IN UDUMALPET TALUK, TIRUPPUR DISTRICT, TAMIL NADU, INDIA
}

\author{
P. Radha*, R. Nagaraj, C. Udhayavani and K. Sivaranjani \\ Siddha Clinical Research Unit, Central Council for Research in Siddha, \\ Ministry of AYUSH, Govt. of India, Palayamkottai, Tirunelveli-627 002, Tamil Nadu, India
}

Keywords: Biodiversity; Floristic; Tamil Nadu; Udumalpet Taluk; Urbanisation.

\begin{abstract}
Survey on the floral diversity is an important activity to assess the existing flora. This study was carried out from December 2017 to December 2018 to document the flora existing in the Udumalpet Taluk, Tiruppur District, Tamil Nadu, India. As a result, a total of 370 taxa belonging to 263 genera of 82 angiosperm families have been documented. $52 \%$ of species of them are herbaceous. Euphorbiaceae and Fabaceae with 23 taxa each, Asteraceae with 21 species, Acanthaceae with 20 species and Amaranthaceae with 18 species are observed as the dominant families. In this article, family, botanical name, habit, vernacular name and various applications of the recorded plants are enumerated systematically. Threats to these plants and possible conservation strategies are also discussed briefly.
\end{abstract}

\section{Introduction}

Flora of a region is considered as an essential part of the environment that determines the wealth of ecosystem and human health (Sandifer et al., 2015). It is highly recommended that, proper utilization of plant resources of a country, state, district, small areas like taluks and villages, has been helpful to maintain the availability and richness of the flora (Gurusamy et al., 2016; Sarvalingam and Rajendran, 2018). Documentation on the flora of rural areas are comparatively less than the checklists of flora on the particular hills, green patches, reserve forests, uphill or otherwise focused on particular group of plants or genus of the family etc. (Singh, 1982; Sinha, 2005; Sukumaran et al., 2008; Mantosh 2013; Vijay and Ashok, 2013; Ganorkar and Kshirsagar, 2013; Kumar and Ritesh, 2014; Anuradha and Murugandam, 2016; Parthipan et al., 2016; Savita and Sanjaykumar, 2017).

Udumalpet Taluk was previously included in the Coimbatore district, after the bifurcation on 2008, it came under newly formed Tiruppur district. Floristic survey of this area was previously done by different researchers mainly on Anamalai Reserve Forests and it's nearby reserve forests, green patches and hillocks coming under this Taluk. However, Thirumoorthy and Amaravathi dams flora was not done extensively (Shankaranarayanan and Gupta, 1959; Balasubramanian et al., 1997; Ramachandran, 2007; George et al., 2011; Rasingam, 2012; Ramachandran et al., 2014; Murugeswaran et al., 2014; Rajendran et al., 2014; Sridharan and Kalpana, 2017). Since the inhabitants of these areas still rely on plants for medicinal, edible and other commercial/miscellaneous uses, the present work focused to explore the flora existing in the rural areas of Udumalpet Taluk by conducting frequent field surveys and assess its conservation status.

*Corresponding author, email: radhasudar@rediffmail.com 


\section{Materials and Methods}

Udumalpet Taluk of Tiruppur District, Tamil Nadu (India) lies between $10^{\circ} 24^{\prime} 23^{\prime \prime} \mathrm{N}$ latitude and $77^{\circ} 24^{\prime} 45^{\prime \prime} \mathrm{E}$ longitude. The average altitude of the study area is $368 \mathrm{~m}$. Annual temperature varies from an average of $20^{\circ} \mathrm{C}$ and $38^{\circ} \mathrm{C}$ and the average annual rainfall is 480 to $600 \mathrm{~mm}$. Total population of the Udumalpet Taluk is 1,29,117 of which 64,600 are male and 64,517 are female from 46 villages. Banana, Coconut, Maize, yellow Pumpkin are the main crops cultivated in the study area.

This area is surrounded by a part of Anamalai hills and two major reservoirs namely, Thirumoorthy Dam and Amaravathi Dam, a part of Indira Gandhi Wildlife Sanctuary and National Park (now Anamalai Tiger Reserve) of Western Ghats crossing Tamil Nadu. Floristic survey of this region was previously done by different researchers mainly on Anamalai Reserve Forests and its nearby reserve forests. No records are found in the Rural floras (George et al., 2011; Rasingam, 2012; Murugeswaran et al., 2014; Rajendran et al., 2014; Sridharan and Kalpana, 2017).

For this study, frequent field explorations were conducted from December 2017 to December 2018 to document the flora existing in the different habitats in the rural areas of Udumalpet Taluk. Specimens were collected in triplicate and identified using various flora (Gamble and Fischer, 1915-1936; Nair and Henry, 1983; Henry et al., 1987, 1989; Chandrabose and Nair, 1987; Matthew, 1981-1983, 1991). The botanical name and vernacular name were verified using reputed websites such as The Plant List (2013), FRLHT etc. The collected plant specimens were processed for herbarium as per the standard herbarium methods (Jain and Rao, 1977) and deposited in the herbaria of Siddha Clinical Research Unit, Palayamkottai for future reference.

\section{Results and Discussion}

A total of 370 taxa belonging to 263 genera spreading over 82 families of Angiosperms along with some species of Pteridophytes such as Selaginella wightii Hieron., Marsilea quadrifolia L., Actinopteris radiata (J. Koenig ex Sw.) Link, Azolla sp. etc., bryophytes such as Marchantia sp., Funararia sp. and a few fungus were documented. In Dicotyledons, the sub-class Polypetalae comprises 38 families of 103 genera with 147 species, Gamopetalae18 families of 99 genera and 134 species, and Monochlamydeae 14 families of 36 genera 63 species, whereas Monocototyledon 12 families of 25 genera with 26 species (Table 1). Each taxon is enumerated with botanical name, family, habit, vernacular name and economic importance such as medicine, edible, ornamental and timber yielding plants (Table 2).

Euphorbiaceae and Fabaceae are dominant with 23 species each followed by Asteraceae (21 species), Acanthaceae (20 species), Amaranthaceae (18 species), Caesalpiniaceae (16 species), Solanaceae (15 species), Malvaceae (13 species), Cucurbitaceae and Mimosaceae (12 species each), Verbenaceae and Lamiaceae (10 species each) and rest of the families comprise less than ten species each (Fig. 1). The habit of the species recorded from the study area is broadly classified under four categories viz., herbs, shrubs, climbers and trees. Herbs constitute the major portion with 196 species (53\%) followed by trees with 82 species (22\%), shrubs with 53 (15\%) and climbers with 38 species (10\%). The tree species such as, Adenanthera pavonina L., Alstonia scholaris (L.) R.Br., Ailanthus excelsa Roxb., Crateva adansonii DC., Dalbergia sissoo Roxb., Delonix elata (L.) Gamble, Delonix regia (Boj. ex Hook) Rafin., Lannea coromandelica (Houtt.) Merr., Millingtonia hortensis L.f., Azadirachta indica A. Juss., Muntingia calabura L., Pongamia pinnata (L.) Pierre, Spathodea campanulata Beauv., Tamarindus indica L., Tectona grandis L.f., Terminalia arjuna (Roxb.) Wight \& Arn., Terminalia catappa L., and Thespesia populnea (L.) Soland ex Correa are recorded from the roadsides/highways. Some of the common species recorded in the study area were given in the Figs 2 and 3. 
Amongst the recorded plants, $332(75.11 \%)$ species are medicinal, $48(10.85 \%)$ species are edible, $30(6.78 \%)$ species are timber yielding, and 27 species $(6.10 \%)$ are ornamentals. Five species are useful in miscellaneous purposes $(1.13 \%)$. The common seasonal herbs of annuals, biennials, climbers and aquatic species are flourished in the studied area that has been used as medicine for common ailments. However, some interesting species such as Caralluma umbellata Haw., Cymbopogon citratus (DC.) Stapf., Dodonaea viscosa (L.) Jacq. and Euphorbia antiquorum L. etc., are found to be dominant along with the other medicinal plants in the small hillocks like Perumalmalai, Thamburanmalai and Perumalpudhur hills.

Species like Ammania baccifera L., Bacopa monnieri (L.) Pennell, Centella asiatica (L.) Urban, Colocasia esculenta (L.) Schott, Commelina benghalensis L., Cyanotis axillaris (L.) D. Don, Eichhornia crassipes (Mart.) Solms-Laub., Pistia stratiotes L., Sphaeranthus amaranthoides Burm.f. and Sphaeranthus indicus L. are recorded from the wetlands. Dendrophthoe falcata (L.f.) Etting. a stem parasite, Striga asiatica (L.) Kuntze and Santalum album L., the root parasites are also found in the study area. Strobilanthes consanguineaus (Nees) T. Anders. is an endemic species of the Southern Western Ghats (Sasidharan, 2004). Santalum album L. (Santalaceae) is recorded as rare during the study period (IUCN, 2018). Eichhornia crassipes (Mart.) Solms-Laub., Lantana camara L. var. aculeata (L.) Mold., Mikania cordata (Burm.f.) B.L.Rob. and Parthenium hysterophorus L. are the exotics species spreading vigorously in the natural habitations and cultivation fields that compete with other important taxa.

Table 1. Numerical representation of the flora of Udumalpet Taluk, Tiruppur District.

\begin{tabular}{lllll}
\hline Class & Sub-class & No. of species & No. of genera & No. of families \\
\hline \multirow{3}{*}{ Dicotyledons } & Polypetalae & 147 & 103 & 38 \\
& Gamopetalae & 134 & 99 & 18 \\
& Monochlamydeae & 63 & 36 & 14 \\
Monocotyledons & - & 26 & 25 & 12 \\
Total & & 370 & 263 & 82 \\
\hline
\end{tabular}

Table 2. List of plants recorded from the study area.

\begin{tabular}{|c|c|c|c|c|c|}
\hline $\begin{array}{l}\text { Sl. } \\
\text { no. }\end{array}$ & Botanical name & Family & Habit & Vernacular name & $\begin{array}{l}\text { Economic } \\
\text { value }\end{array}$ \\
\hline 1. & Abrus precatorius $\mathrm{L}$. & Fabaceae & $\mathrm{Cl}$ & Kuntumani & $\mathrm{M}$ \\
\hline 2. & Abutilon crispum (L.) Medicus & Malvaceae & $\mathrm{H}$ & Siruthuthi & - \\
\hline 3. & Abutilon hirtum (Lam.) Sweet & Malvaceae & $\mathrm{S}$ & Vattathuthi & M \\
\hline 4. & Abutilon indicum (L.) Sweet & Malvaceae & $S$ & Thuthi & M \\
\hline 5. & Acacia horrida (L.f.) Willd. & Mimosaceae & $\mathrm{T}$ & Anaimullu & - \\
\hline 6. & Acacia leucophloea (Roxb.) Willd. & Mimosaceae & $\mathrm{T}$ & Velvelam & $\mathrm{M} / \mathrm{Ti}$ \\
\hline 7. & $\begin{array}{l}\text { Acacia nilotica (L.) Willd. ex Del. } \\
\text { subsp. indica (Benth) Brenan }\end{array}$ & Mimosaceae & $\mathrm{T}$ & Karuvelam & $\mathrm{M} / \mathrm{Ti}$ \\
\hline 8. & Acacia planifrons Wight \& Arn. & Mimosaceae & $\mathrm{T}$ & Kodaivelam & $\mathrm{M} / \mathrm{Ti}$ \\
\hline 9. & Acalypha fruticosa Forssk. & Euphorbiaceae & $\mathrm{H}$ & Sinni & M \\
\hline 10. & Acalypha indica $\mathrm{L}$. & Euphorbiaceae & $\mathrm{H}$ & Kuppaimeni & M \\
\hline 11. & Acalypha racemosa Heyne ex Baill. & Euphorbiaceae & $\mathrm{H}$ & Orvisakarappan & M \\
\hline 12. & Acanthospermum hispidum DC. & Asteraceae & $\mathrm{H}$ & Mutthulasi & M \\
\hline
\end{tabular}




\begin{tabular}{|c|c|c|c|c|c|}
\hline 13. & Achyranthes aspera $\mathrm{L}$. & Amaranthaceae & $\mathrm{H}$ & Nayurivi & $\mathrm{M}$ \\
\hline 14. & Achyranthes bidentata Blume & Amaranthaceae & $\mathrm{H}$ & Sennaiyuruvi & M \\
\hline 15. & Adenanthera pavonina $\mathrm{L}$. & Mimosaceae & $\mathrm{T}$ & Aanai Kundumani & M \\
\hline 16. & Aegle marmelos (L.) Correa & Rutaceae & $\mathrm{T}$ & Vilvam & M \\
\hline 17. & $\begin{array}{l}\text { Aerva javanica (Burm.f.) Juss. ex } \\
\text { Schultes }\end{array}$ & Amaranthaceae & $\mathrm{H}$ & Perumpeelai & M \\
\hline 18. & Aerva lanata (L.) Juss. ex Schultes & Amaranthaceae & $\mathrm{H}$ & Sirupeelai & M \\
\hline 19. & Agave americana $\mathrm{L}$. & Agavaceae & $\mathrm{S}$ & Kathazhai & $\mathrm{M} / \mathrm{O}$ \\
\hline 20. & Ageratum conyzoides $\mathrm{L}$. & Asteraceae & $\mathrm{H}$ & Pumpillu & M \\
\hline 21. & Ailanthus excelsa Roxb. & Simaroubaceae & $\mathrm{T}$ & Perumaram & $\mathrm{M} / \mathrm{Ti}$ \\
\hline 22. & Albizia amara (Roxb.) Boivin & Mimosaceae & $\mathrm{T}$ & Arappu & M \\
\hline 23. & Albizia lebbeck (L.) Willd. & Mimosaceae & $\mathrm{T}$ & Vagai & $\mathrm{M} / \mathrm{Ti}$ \\
\hline 24. & Albizia saman (Jacq.) F.v. Muell. & Mimosaceae & $\mathrm{T}$ & Thoongu moonji & $\mathrm{M} / \mathrm{Ti}$ \\
\hline 25. & Allamanda cathartica $\mathrm{L}$. & Apocynaceae & $\mathrm{Cl}$ & Manjal-patti & $\mathrm{M} / \mathrm{O}$ \\
\hline 26. & Allmania nodiflora (L.) R. Br. ex Wight & Amaranthaceae & $\mathrm{H}$ & Kummatikeerai & $\mathrm{M} / \mathrm{E}$ \\
\hline 27. & Aloe vera (L.) Burm.f. & Lilliaceae & $\mathrm{H}$ & Chotthukathazhai & $\mathrm{M}$ \\
\hline 28. & Alstonia scholaris (L.) R.Br. & Apocynaceae & $\mathrm{T}$ & Yezhilai paalai & M \\
\hline 29. & $\begin{array}{l}\text { Alternanthera philoxeroides (C. Martiu) } \\
\text { Griseb. }\end{array}$ & Amaranthaceae & $\mathrm{H}$ & - & $\mathrm{M} / \mathrm{E}$ \\
\hline 30. & Alternanthera pungens Kunth & Amaranthaceae & $\mathrm{H}$ & Adai otti & M \\
\hline 31. & Alternanthera sessilis (L.) R.Br. ex DC. & Amaranthaceae & $\mathrm{H}$ & Ponnanganni & $\mathrm{M} / \mathrm{E}$ \\
\hline 32. & Alternanthera tenella Colla. & Amaranthaceae & $\mathrm{H}$ & - & $\mathrm{M} / \mathrm{E}$ \\
\hline 33. & Alysicarpus glumaceus (Vahl) DC. & Fabaceae & $\mathrm{H}$ & - & M \\
\hline 34. & Alysicarpus vaginalis (L.) DC. & Fabaceae & $\mathrm{H}$ & Kuruthiadakki & M \\
\hline 35. & Amaranthus polygonoides $\mathrm{L}$. & Amaranthaceae & $\mathrm{H}$ & Sirukeerai & $\mathrm{E}$ \\
\hline 36. & Amaranthus spinosus $\mathrm{L}$. & Amaranthaceae & $\mathrm{H}$ & Mullukkeerai & $\mathrm{M} / \mathrm{E}$ \\
\hline 37. & Amaranthus tricolor $\mathrm{L}$. & Amaranthaceae & $\mathrm{H}$ & Thandukeerai & $\mathrm{M} / \mathrm{E}$ \\
\hline 38. & Amaranthus viridis $\mathrm{L}$. & Amaranthaceae & $\mathrm{H}$ & Kupaikeerai & $\mathrm{E}$ \\
\hline 39. & Ammania baccifera $\mathrm{L}$. & Lythraceae & $\mathrm{H}$ & Neermel-neruppu & M \\
\hline 40. & Anaphalis subdecurrens (DC.) Gamble & Asteraceae & $\mathrm{H}$ & - & - \\
\hline 41. & Andrographis echioides Nees & Acanthaceae & $\mathrm{H}$ & Gopuram thaangi & M \\
\hline 42. & $\begin{array}{l}\text { Andrographis paniculata (Burm.f.) } \\
\text { Wall. ex Nees }\end{array}$ & Acanthaceae & $\mathrm{H}$ & Siriyanangai & M \\
\hline 43. & $\begin{array}{l}\text { Anisochilus carnosus (L.f.) Wall. ex } \\
\text { Benth. }\end{array}$ & Lamiaceae & $\mathrm{H}$ & Poochenthira pattai & M \\
\hline 44. & $\begin{array}{l}\text { Anisomeles malabarica (L.) R. Br. ex } \\
\text { Sims }\end{array}$ & Lamiaceae & $\mathrm{H}$ & Peimiratti & M \\
\hline 45. & Annona squamosa $\mathrm{L}$ & Annonaceae & $\mathrm{T}$ & Seetha & $\mathrm{E}$ \\
\hline 46. & Antigonon leptopus Hook. \& Arn. & Polygonaceae & $\mathrm{Cl}$ & Kodi rose & $\mathrm{O}$ \\
\hline 47. & Argemone mexicana $\mathrm{L}$. & Papaveraceae & $\mathrm{H}$ & Bramathandu & M \\
\hline 48. & Aristolochia bracteolata Lam. & Aristolochiaceae & $\mathrm{H}$ & Aduthinnappalai & M \\
\hline 49. & Aristolochia indica $\mathrm{L}$. & Aristolochiaceae & $\mathrm{Cl}$ & Esuramooli & M \\
\hline 50. & Artocarpus heterophyllus Lam. & Moraceae & $\mathrm{T}$ & Palamaram & $\mathrm{E} / \mathrm{Ti}$ \\
\hline 51. & Arundo donax $\mathrm{L}$ & Poaceae & $\mathrm{H}$ & Southai moongil & $\mathrm{O}$ \\
\hline 52. & Asparagus racemosus Willd. & Lilliaceae & $\mathrm{H}$ & $\begin{array}{l}\text { Thaneer vitaan } \\
\text { kizhangu }\end{array}$ & M \\
\hline 53. & Asystasia gangetica (L.) T. And. & Acanthaceae & $\mathrm{H}$ & Silathinayagam & M \\
\hline 54. & Azadirachta indica A. Juss. & Meliaceae & $\mathrm{T}$ & Vembu & $\mathrm{M} / \mathrm{Ti}$ \\
\hline
\end{tabular}




\begin{tabular}{|c|c|c|c|c|c|}
\hline 55. & Azima tetracantha Lam. & Salvadoraceae & $S$ & Mulsangu & M \\
\hline 56. & Bacopa monnieri (L.) Pennell & Scrophulariaceae & $\mathrm{H}$ & Neerbrahmi & M \\
\hline 57. & Barleria acuminata Nees & Acanthaceae & $\mathrm{H}$ & Vellai kurinji & M \\
\hline 58. & Barleria buxifolia $\mathrm{L}$. & Acanthaceae & $\mathrm{S}$ & Chunampu korandi & M \\
\hline 59. & Barleria cuspidata Heyne ex Nees & Acanthaceae & $\mathrm{H}$ & Manjal semmuli & M \\
\hline 60. & Barleria prionitis $\mathrm{L}$ & Acanthaceae & $\mathrm{H}$ & Chemmulli & M \\
\hline 61. & Basella rubra $\mathrm{L}$. & Basellaceae & $\mathrm{H}$ & Pasalai Keerai & $\mathrm{M} / \mathrm{E}$ \\
\hline 62. & Bauhinia racemosa Lam. & Caesalpiniaceae & $\mathrm{T}$ & Aathi & $\mathrm{M} / \mathrm{Ti}$ \\
\hline 63. & Bauhinia tomentosa $\mathrm{L}$. & Caesalpiniaceae & $\mathrm{T}$ & Iruvatchi & M \\
\hline 64. & $\begin{array}{l}\text { Benkara malabarica (Lam.) } \\
\text { Tirvengadum }\end{array}$ & Rubiaceae & $\mathrm{T}$ & Matukkarai & M \\
\hline 65. & Blainvillea acmella (L.) Philipson & Asteraceae & $\mathrm{H}$ & - & M \\
\hline 66. & $\begin{array}{l}\text { Blepharis maderaspatensis (L.) Heyne } \\
\text { ex Roth }\end{array}$ & Acanthaceae & $\mathrm{H}$ & Nethira moolli & M \\
\hline 67. & Blepharis molluginifolia Pers. & Acanthaceae & $\mathrm{H}$ & - & M \\
\hline 68. & $\begin{array}{l}\text { Boerhavia chinensis (L.) Asch. \& } \\
\text { Schweinf. }\end{array}$ & Nyctaginaceae & $\mathrm{H}$ & Sambal poondu & M \\
\hline 69. & Boerhavia diffusa $\mathrm{L}$. & Nyctaginaceae & $\mathrm{H}$ & Mookarattai & M \\
\hline 70. & Boerhavia erecta $\mathrm{L}$. & Nyctaginaceae & $\mathrm{H}$ & Seemai mookarattai & M \\
\hline 71. & Borassus flabellifer $\mathrm{L}$. & Arecaceae & $\mathrm{T}$ & Panaimaram & $\mathrm{M} / \mathrm{E} / \mathrm{Ti}$ \\
\hline 72. & Bougainvillea glabra Choisy & Nyctaginaceae & S & Kaagitha poo & $\mathrm{O}$ \\
\hline 73. & Brassica juncea (L.) Czern. \& Coss. & Apiaceae & $\mathrm{H}$ & Kadugu & $\mathrm{M} / \mathrm{E}$ \\
\hline 74. & Byttneria herbacea Roxb. & Sterculiaceae & $\mathrm{H}$ & - & M \\
\hline 75. & Caesalpinia bonduc (L.) Roxb. & Caesalpiniaceae & $\mathrm{S}$ & Kazharchikaai & M \\
\hline 76. & Caesalpinia pulcherrima (L.) Sw. & Caesalpiniaceae & $\mathrm{S}$ & Mayil kondrai & $\mathrm{M} / \mathrm{O}$ \\
\hline 77. & Calotropis gigantea (L.) R.Br. & Asclepiadaceae & $S$ & Erukku & M \\
\hline 78. & Calotropis procera (Ait.) R.Br. & Asclepiadaceae & $\mathrm{S}$ & Vellerukku & M \\
\hline 79. & $\begin{array}{l}\text { Canthium coromandelicum (Burm. f.) } \\
\text { Alston }\end{array}$ & Rubiaceae & $\mathrm{S}$ & Kaaraichedi & M \\
\hline 80. & Capparis divaricata Lam. & Capparidiaceae & $S$ & Thoratti & M \\
\hline 81. & Capparis sepiaria $\mathrm{L}$. & Capparidaceae & S & Karunjurai & M \\
\hline 82. & Capparis zeylanica $\mathrm{L}$. & Capparidaceae & $S$ & Athondai & M \\
\hline 83. & Capsicum annuит $\mathrm{L}$. & Solanaceae & $\mathrm{H}$ & Milagai & $\mathrm{M} / \mathrm{E}$ \\
\hline 84. & Caralluma adscendens (Roxb.) Haw. & Asclepiadaceae & $\mathrm{H}$ & Kallimulayan & M \\
\hline 85. & Caralluma umbellata Haw. & Asclepiadaceae & $\mathrm{H}$ & Yaanai Kallimuliyaan & M \\
\hline 86. & Cardiospermum canescens Wall. & Sapindaceae & $\mathrm{Cl}$ & $\begin{array}{l}\text { Kaattu } \\
\text { mudakkaruthaan }\end{array}$ & M \\
\hline 87. & Cardiospermum halicacabum $\mathrm{L}$. & Sapindaceae & $\mathrm{Cl}$ & Mudakotthan & M \\
\hline 88. & Carica papaya $\mathrm{L}$ & Caricaceae & $\mathrm{T}$ & Pappali & $\mathrm{M} / \mathrm{E}$ \\
\hline 89. & Caryota urens $\mathrm{L}$. & Arecaceae & $\mathrm{T}$ & Koonthalpanai & $\mathrm{M} / \mathrm{O}$ \\
\hline 90. & Cassia auriculata $\mathrm{L}$. & Caesalpiniaceae & S & Avaram & M \\
\hline 91. & Cassia hirsuta (L.) Irwin \& Barneby & Caesalpiniaceae & $\mathrm{H}$ & Malaiyavarai & M \\
\hline 92. & Cassia italica Mill. & Caesalpiniaceae & $\mathrm{H}$ & Nilvagai & M \\
\hline 93. & Cassia roxburghii $\mathrm{DC}$. & Caesalpiniaceae & $\mathrm{T}$ & Senkondrai & $\mathrm{O}$ \\
\hline 94. & Catharanthus pusillus (Murr.) G.Don & Apocynaceae & $\mathrm{H}$ & Milagai poondu & M \\
\hline 95. & Catharanthus roseus (L.) G.Don & Apocynaceae & $\mathrm{H}$ & Nithyakalyani & $\mathrm{M} / \mathrm{O}$ \\
\hline 96. & Ceiba pentandra (L.) Gaertn. & Bombacaceae & $\mathrm{T}$ & Ilavam & M \\
\hline 97. & Celosia argentea $\mathrm{L}$. & Amaranthaceae & $\mathrm{H}$ & Pannaikeerai & $\mathrm{M} / \mathrm{E}$ \\
\hline
\end{tabular}




\begin{tabular}{|c|c|c|c|c|c|}
\hline 98. & Centella asiatica (L.) Urban & Apiaceae & $\mathrm{H}$ & Vallarai & $\mathrm{M} / \mathrm{E}$ \\
\hline 99. & Cereus pterogonus Lem. & Cactaceae & $\mathrm{S}$ & Sippaikalli & - \\
\hline 100. & Ceropegia juncea Roxb. & Asclepiadaceae & $\mathrm{Cl}$ & Pulichakodi & M \\
\hline 101. & $\begin{array}{l}\text { Chamaecrista absus (L.) H. Irwin \& } \\
\text { Barneby }\end{array}$ & Caesalpiniaceae & $\mathrm{H}$ & Mulaipalyirai & M \\
\hline 102. & Chenopodium ambrosioides L. & Chenopodiaceae & $\mathrm{H}$ & Mannenaikolai & M \\
\hline 103. & Chloris barbata $\mathrm{Sw}$. & Poaceae & $\mathrm{H}$ & Kodai pillu & M \\
\hline 104. & $\begin{array}{l}\text { Chromolaena odorata (L.) King \& } \\
\text { Robinson }\end{array}$ & Asteraceae & $\mathrm{S}$ & - & M \\
\hline 105. & Cissus quadrangularis $\mathrm{L}$. & Vitaceae & $\mathrm{Cl}$ & Pirandai & $\mathrm{M} / \mathrm{E}$ \\
\hline 106. & Citrullus colocynthis (L.) Schrader & Cucurbitaceae & $\mathrm{H}$ & Peikkumatti & M \\
\hline 107. & $\begin{array}{l}\text { Citrullus lanatus (Thunb.) Matsumura } \\
\& \text { Nakai }\end{array}$ & Cucurbitaceae & $\mathrm{Cl}$ & Kattu thannipalam & M \\
\hline 108. & Citrus limon (L.) Burm.f. & Rutaceae & $\mathrm{T}$ & Elumichai & $\mathrm{M} / \mathrm{E}$ \\
\hline 109. & Citrus medica $\mathrm{L}$. & Rutaceae & $\mathrm{T}$ & Narathai & $\mathrm{M} / \mathrm{E}$ \\
\hline 110. & Cleome gynandra $\mathrm{L}$. & Capparidaceae & $\mathrm{H}$ & Nalvaelai & M \\
\hline 111. & Cleome monophylla $\mathrm{L}$. & Capparidaceae & $\mathrm{H}$ & Ucivaelai & M \\
\hline 112. & Cleome viscosa $\mathrm{L}$. & Capparidaceae & $\mathrm{H}$ & Nai kadugu & M \\
\hline 113. & Clerodendrum phlomidis L.f. & Verbenaceae & S & Thazhuthaazhai & M \\
\hline 114. & Clitoria ternatea $\mathrm{L}$. & Fabaceae & $\mathrm{Cl}$ & Sangu poo & $\mathrm{M} / \mathrm{O}$ \\
\hline 115. & Coccinia grandis (L.) Voigt & Cucurbitaceae & $\mathrm{Cl}$ & Kovai & $\mathrm{M} / \mathrm{E}$ \\
\hline 116. & Cocculus hirsutus (L.) Diels & Menispermaceae & $\mathrm{Cl}$ & Kattukodi & M \\
\hline 117. & Cocos nucifera $\mathrm{L}$ & Arecaceae & $\mathrm{T}$ & Thenneimaram & $\mathrm{M} / \mathrm{E} / \mathrm{Ti}$ \\
\hline 118. & Coldenia procumbens $\mathrm{L}$. & Boraginaceae & $\mathrm{H}$ & Seruppadai & M \\
\hline 119. & Colocasia esculenta (L.) Schott & Araceae & $\mathrm{H}$ & Saeppan kizhangu & $\mathrm{E}$ \\
\hline 120. & Commelina benghalensis L. & Commelinaecae & $\mathrm{H}$ & Kanavazhai & $\mathrm{E}$ \\
\hline 121. & Commelina diffusa Burm & Commelinaceae & $\mathrm{H}$ & - & M \\
\hline 122. & Commiphora berryi (Arn.) Engler & Burseraceae & $\mathrm{T}$ & Mullu kiluvai & M \\
\hline 123. & $\begin{array}{l}\text { Corallocarpus epigaeus (Rottl. \& } \\
\text { Willd.) Clarke }\end{array}$ & Cucurbitaceae & $\mathrm{Cl}$ & Garudan kizhangu & M \\
\hline 124. & Corchorus trilocularis $\mathrm{L}$. & Tiliaceae & $\mathrm{H}$ & Vazhukkai poondu & M \\
\hline 125. & Cordia sebestena $\mathrm{L}$. & Boraginaceae & $\mathrm{T}$ & - & $\mathrm{M} / \mathrm{O}$ \\
\hline 126. & Coriandrum sativum $\mathrm{L}$. & Apiaceae & $\mathrm{H}$ & Kothamalli & $\mathrm{M} / \mathrm{E}$ \\
\hline 127. & Couroupita guianensis Aubl. & Lecythidaceae & $\mathrm{T}$ & Nagalingam & M \\
\hline 128. & Crateva adansonii DC. & Capparidaceae & $\mathrm{T}$ & Mavilingam & $\mathrm{M} / \mathrm{Ti}$ \\
\hline 129. & Crinum asiaticum $\mathrm{L}$. & Amaryllidaceae & $\mathrm{H}$ & Vishamoongil & M \\
\hline 130. & Crossandra infundibuliformis (L.) Nees & Acanthaceae & $\mathrm{H}$ & Kanakambaram & $\mathrm{M} / \mathrm{O}$ \\
\hline 131. & Crotalaria globosa Wight \& Arn. & Fabaceae & $\mathrm{H}$ & - & - \\
\hline 132. & Crotalaria pallida Dryand. & Fabaceae & $\mathrm{H}$ & - & M \\
\hline 133. & $\begin{array}{l}\text { Crotalaria pallida Dryand. var. obovata } \\
\text { (G.Don) Polhill }\end{array}$ & Fabaceae & $\mathrm{H}$ & - & - \\
\hline 134. & Crotalaria verrucosa $\mathrm{L}$. & Fabaceae & $\mathrm{H}$ & Kilukilupai & $\mathrm{M} / \mathrm{O}$ \\
\hline 135. & Croton bonplandianum Baill. & Euphorbiaceae & $\mathrm{H}$ & Rail poondu & M \\
\hline 136. & Ctenolepis garcinii (Burm.f.) Clarke & Cucurbitaceae & $\mathrm{Cl}$ & Kollankovai & M \\
\hline 137. & Cucumis dipsaceus Ehrenb. ex Spach. & Cucurbitaceae & $\mathrm{Cl}$ & - & M \\
\hline
\end{tabular}




\begin{tabular}{|c|c|c|c|c|c|}
\hline 138. & Cucurbita maxima Duchesne ex Lam. & Cucurbitaceae & $\mathrm{Cl}$ & Parangikaai & M \\
\hline 139. & Cuscuta reflexa Lam. & Convolvulaceae & $\mathrm{Cl}$ & Ammaiyar kuntal & M \\
\hline 140. & Cyanotis axillaris (L.) D. Don & Commelinaceae & $\mathrm{H}$ & Valukkai pullu & M \\
\hline 141. & $\begin{array}{l}\text { Cylindropuntia ramosissima (Engler) } \\
\text { Knuth }\end{array}$ & Cactaceae & $\mathrm{S}$ & Uruttai Chapathikalli & Mis. \\
\hline 142. & Cymbopogon citratus (DC.) Stapf & Poaceae & $\mathrm{H}$ & Vasana pullu & M \\
\hline 143. & Cynodon dactylon (L.) Pers. & Poaceae & $\mathrm{H}$ & Arugam pullu & M \\
\hline 144. & Dactyloctenium aegyptium (L.) Willd. & Poaceae & $\mathrm{H}$ & Aricipul & M \\
\hline 145. & Dalbergia sissoo Roxb. & Fabaceae & $\mathrm{T}$ & Sisu & $\mathrm{M} / \mathrm{Ti}$ \\
\hline 146. & Datura innoxia Mill. & Solanaceae & $\mathrm{H}$ & Oomatthai & M \\
\hline 147. & Datura metel L. & Solanaceae & $\mathrm{H}$ & Karu Oomatthai & M \\
\hline 148. & Delonix elata (L.) Gamble & Caesalpiniaceae & $\mathrm{T}$ & Vaadhanarayanan & M \\
\hline 149. & Delonix regia (Boj. ex Hook) Rafin. & Caesalpiniaceae & $\mathrm{T}$ & Mayil Kondrai & $\mathrm{M} / \mathrm{Ti}$ \\
\hline 150. & Dendrophthoe falcata (L.f.) Etting. & Loranthaceae & S & Pulluruvi & M \\
\hline 151. & $\begin{array}{l}\text { Dichrostachys cinerea }(\mathrm{L} .) \text { Wight \& } \\
\text { Arn. }\end{array}$ & Mimosaceae & $\mathrm{T}$ & Vidathazhai & $\mathrm{M} / \mathrm{Ti}$ \\
\hline 152. & Dicoma tomentosa Cass. & Asteraceae & $\mathrm{H}$ & - & M \\
\hline 153. & Diplocyclos palmatus (L.) Jeffrey & Cucurbitaceae & $\mathrm{Cl}$ & Iyvrali & M \\
\hline 154. & Dipteracanthus prostratus (Poir.) Nees & Acanthaceae & $\mathrm{H}$ & Pottakanchi & M \\
\hline 155. & Dodonaea viscosa (L.) Jacq. & Sapindaceae & $\mathrm{S}$ & Viraali & M \\
\hline 156. & $\begin{array}{l}\text { Dolichandrone atrovirens (Heyne } e x \\
\text { Roth) Sprague }\end{array}$ & Bignoniaceae & $\mathrm{T}$ & - & $\mathrm{M} / \mathrm{O}$ \\
\hline 157. & Echinochloa colona $(\mathrm{L}$.$) Link$ & Poaceae & $\mathrm{H}$ & Karumpullu & M \\
\hline 158. & Eclipta prostrata $(\mathrm{L}.) \mathrm{L}$. & Asteraceae & $\mathrm{H}$ & Vellai karisalankanni & M \\
\hline 159. & $\begin{array}{l}\text { Eichhornia crassipes (Mart.) Solms- } \\
\text { Laub. }\end{array}$ & Pontederiaceae & $\mathrm{H}$ & Vengaaya thamarai & M \\
\hline 160. & Enicostema axillare (Lam.) Raynal & Gentianaceae & $\mathrm{H}$ & Vellaragu & M \\
\hline 161. & Eragrostiella bifaria (Vahl) Bor & Poaceae & $\mathrm{H}$ & Oothupul & Mis. \\
\hline 162. & $\begin{array}{l}\text { Eragrostis unioloides (Retz.) Nees ex } \\
\text { Steud. }\end{array}$ & Poaceae & $\mathrm{H}$ & - & - \\
\hline 163. & Euphorbia antiquorum $\mathrm{L}$. & Euphorbiaceae & $\mathrm{S}$ & Sadura-kalli & M \\
\hline 164. & Euphorbia cyathophora Murr. & Euphorbiaceae & $\mathrm{H}$ & Poinsettia & $\mathrm{O}$ \\
\hline 165. & Euphorbia heterophylla $\mathrm{L}$. & Euphorbiaceae & $\mathrm{H}$ & Paal poondu chedi & $\mathrm{E}$ \\
\hline 166. & Euphorbia hirta $\mathrm{L}$. & Euphorbiaceae & $\mathrm{H}$ & Ammanpacharisi & M \\
\hline 167. & Euphorbia thymifolia $\mathrm{L}$. & Euphorbiaceae & $\mathrm{H}$ & Sittrapaladai & M \\
\hline 168. & Euphorbia tirucalli $\mathrm{L}$. & Euphorbiaceae & $\mathrm{S}$ & Tiru-kalli & $\mathrm{M} / \mathrm{O}$ \\
\hline 169. & Euphorbia tortilis Rottl. & Euphorbiaceae & $\mathrm{S}$ & Thirugukalli & M \\
\hline 170. & Evolvulus alsinoides $(\mathrm{L}.) \mathrm{L}$ & Convolvulaceae & $\mathrm{H}$ & Vishnukarandi & M \\
\hline 171. & Ficus auriculata Lour. & Moraceae & $\mathrm{T}$ & - & $\mathrm{M} / \mathrm{O}$ \\
\hline 172. & Ficus benghalensis $\mathrm{L}$. & Moraceae & $\mathrm{T}$ & Aal & M \\
\hline 173. & Ficus religiosa $\mathrm{L}$. & Moraceae & $\mathrm{T}$ & Arasamaram & $\mathrm{M} / \mathrm{Ti}$ \\
\hline 174. & Glinus lotoides L. & Aizoaceae & $\mathrm{H}$ & Siruseruppadai & M \\
\hline 175. & Glinus oppositifolius (L.) A. DC. & Aizoaceae & $\mathrm{H}$ & Katchanthura & M \\
\hline 176. & Gmelina arborea Roxb. & Verbenaceae & $\mathrm{T}$ & Kumizha maram & $\mathrm{M} / \mathrm{O}$ \\
\hline 177. & Gmelina asiatica $\mathrm{L}$. & Verbenaceae & $\mathrm{T}$ & Nilakkumizh & M \\
\hline 178. & Gomphrena celosioides C. Martius & Amaranthaceae & $\mathrm{H}$ & Neer vadamalli & $\mathrm{E}$ \\
\hline 179. & Gomphrena globosa $\mathrm{L}$. & Amaranthaceae & $\mathrm{H}$ & Vaadamalli & $\mathrm{M} / \mathrm{O}$ \\
\hline 180. & Gyrocarpus americanus Willd. & Hernandiaceae & $\mathrm{T}$ & Thanakku & $\mathrm{Ti}$ \\
\hline
\end{tabular}




\begin{tabular}{|c|c|c|c|c|c|}
\hline 181. & Hamelia patens Jacq. & Rubiaceae & $\mathrm{S}$ & - & $\mathrm{M} / \mathrm{O} / \mathrm{E}$ \\
\hline 182. & Hardwickia binata Roxb. & Caesalpiniaceae & $\mathrm{T}$ & Acha maram & $\mathrm{O} / \mathrm{Ti}$ \\
\hline 183. & Heliotropium indicum $\mathrm{L}$. & Boraginaceae & $\mathrm{H}$ & Thael kodukku & M \\
\hline 184. & Heliotropium scabrum Retz. & Boraginaceae & $\mathrm{H}$ & - & - \\
\hline 185. & Hemidesmus indicus (L.) R.Br. & Asclepiadaceae & $\mathrm{Cl}$ & Nannaari & $\mathrm{M} / \mathrm{E}$ \\
\hline 186. & Hibiscus lobatus (Murr.) Kuntze & Malvaceae & $\mathrm{H}$ & - & - \\
\hline 187. & Hibiscus micranthus L.f. & Malvaceae & $\mathrm{H}$ & Sitraamutti & M \\
\hline 188. & Hibiscus vitifolius $\mathrm{L}$. & Malvaceae & S & Siru thutthi & M \\
\hline 189. & Holoptelea integrifolia (Roxb.) Planch. & Ulmaceae & $\mathrm{T}$ & Aya & $\mathrm{Ti}$ \\
\hline 190. & Homonoia riparia Lour. & Euphorbiaceae & $S$ & Kallarali & M \\
\hline 191. & $\begin{array}{l}\text { Hybanthus enneaspermus (L.) F. V. } \\
\text { Muell. }\end{array}$ & Violaceae & $\mathrm{H}$ & Oridhal thamarai & M \\
\hline 192. & $\begin{array}{l}\text { Hygrophila schulli (Hamilt.) } \\
\text { M.R.Almeida \& S.M. Almeida }\end{array}$ & Acanthaceae & $\mathrm{H}$ & Neermulli & M \\
\hline 193. & Hyptis suaveolens (L.) Poit. & Lamiaceae & $\mathrm{H}$ & Kanathulasi & M \\
\hline 194. & Imperata cylindrica (L.) Raeusch. & Poaceae & $\mathrm{H}$ & Dharbai pullu & M \\
\hline 195. & Indigofera linnaei $\mathrm{Ali}$ & Fabaceae & $\mathrm{H}$ & Cheppunerunjil & M \\
\hline 196. & Indigofera longiracemosa Boiv. & Fabaceae & $\mathrm{H}$ & - & - \\
\hline 197. & Indigofera tinctoria $\mathrm{L}$. & Fabaceae & $\mathrm{H}$ & Avuri & M \\
\hline 198. & Ipomoea carnea Jacq. & Convolvulaceae & S & Velikaththan & M \\
\hline 199. & Ipomoea hederifolia $\mathrm{L}$. & Convolvulaceae & $\mathrm{Cl}$ & Kanavalikkodi & M \\
\hline 200. & Ipomoea obscura (L.) Ker-Gawl. & Convolvulaceae & $\mathrm{Cl}$ & Siru thali & M \\
\hline 201. & Ipomoea sepiaria Koen. & Convolvulaceae & $\mathrm{Cl}$ & Talikeerai & M \\
\hline 202. & Ipomoea staphylina Roem. \& Schultes & Convolvulaceae & $\mathrm{Cl}$ & Onaan kodi & M \\
\hline 203. & Ixora coccinea $\mathrm{L}$ & Rubiaceae & S & Vetchi & M \\
\hline 204. & Jatropha curcas $\mathrm{L}$. & Euphorbiaceae & S & Kaatu-amanakku & M \\
\hline 205. & Jatropha glandulifera Roxb. & Euphorbiaceae & S & Vellai adalai & M \\
\hline 206. & Jatropha gossypifolia $\mathrm{L}$. & Euphorbiaceae & S & Kaatu-amanakku & M \\
\hline 207. & Justicia adhatoda L. & Acanthaceae & $\mathrm{S}$ & Adathodai & M \\
\hline 208. & Justicia tranquebariensis L.f. & Acanthaceae & $\mathrm{H}$ & Thavasi murungai & M \\
\hline 209. & Kedrostis foetidissima (Jacq.) Cogn. & Cucurbitaceae & $\mathrm{Cl}$ & Appakovai & M \\
\hline 210. & Kylinga bulbosa P. Beavu. & Cyperaceae & $\mathrm{H}$ & - & M \\
\hline 211. & Lablab purpureus (L.) Sweet & Fabaceae & $\mathrm{Cl}$ & Avarai & $\mathrm{E}$ \\
\hline 212. & Lannea coromandelica (Houtt.) Merr. & Anacardiaceae & $\mathrm{T}$ & Odhiya maram & $\mathrm{M} / \mathrm{Ti}$ \\
\hline 213. & $\begin{array}{l}\text { Lantana camara L. var. aculeata (L.) } \\
\text { Mold. }\end{array}$ & Verbenaceae & $S$ & Unnichedi & M \\
\hline 214. & Lantana camera $\mathrm{L}$ & Verbenaceae & S & Unnichedi & M \\
\hline 215. & Lawsonia inermis L. & Lythraceae & $\mathrm{S}$ & Marudhaani & M \\
\hline 216. & Leonotis nepetiifolia (L.) R.Br. & Lamiaceae & $\mathrm{H}$ & Murandai & M \\
\hline 217. & Lepidagathis fasciculata (Retz.) Nees & Acanthaceae & $\mathrm{H}$ & - & - \\
\hline 218. & Lepidagathis pungens Nees & Acanthaceae & $\mathrm{H}$ & Parkurandi & M \\
\hline 219. & Lepidagathis scariosa Nees & Acanthaceae & $\mathrm{H}$ & Sadaikurandi & M \\
\hline 220. & Leucaena leucocephala (L.) Gills & Mimosaceae & $\mathrm{T}$ & Soundil & Mis \\
\hline 221. & Leucas aspera (Willd.) Link & Lamiaceae & $\mathrm{H}$ & Thumbai & M \\
\hline 222. & Ludwigia perennis $\mathrm{L}$. & Onagraceae & $\mathrm{H}$ & Musalkathilai & M \\
\hline 223. & Ludwigia peruviana (L.) Hara & Onagraceae & S & - & M \\
\hline 224. & Luffa cylindrica (L.) M.Roem. & Cucurbitaceae & $\mathrm{Cl}$ & Mozhukupirkanakai & M \\
\hline 225. & Mangifera indica $\mathrm{L}$. & Anacardiaceae & $\mathrm{T}$ & Maa maram & $\mathrm{M} / \mathrm{E}$ \\
\hline
\end{tabular}




\begin{tabular}{|c|c|c|c|c|c|}
\hline 226. & Martynia annua $\mathrm{L}$. & Pedaliaceae & $\mathrm{H}$ & Thael Kodukku & M \\
\hline 227. & Melochia corchorifolia $\mathrm{L}$. & Sterculiaceae & $\mathrm{H}$ & Punnakku poondu & $\mathrm{M}$ \\
\hline 228. & Merremia tridentata (L.) Hall.f. & Convolvulaceae & $\mathrm{H}$ & Avvaiyar koondhal & M \\
\hline 229. & $\begin{array}{l}\text { Microstachys chamaelea (L.) Muell.- } \\
\text { Arg. }\end{array}$ & Euphorbiaceae & $\mathrm{H}$ & Kuruvikachedi & - \\
\hline 230. & Mikania cordata (Burm.f.) B.L.Rob. & Asteraceae & $\mathrm{Cl}$ & - & M \\
\hline 231. & Millingtonia hortensis L.f. & Bignoniaceae & $\mathrm{T}$ & Maramalli & $\mathrm{M} / \mathrm{O}$ \\
\hline 232. & Mimosa pudica $\mathrm{L}$. & Mimosaceae & $\mathrm{H}$ & Thotaal surungi & M \\
\hline 233. & Mimusops elengi $\mathrm{L}$. & Sapotaceae & $\mathrm{T}$ & Mahilam & $\mathrm{M} / \mathrm{O}$ \\
\hline 234. & Mirabilis jalapa $\mathrm{L}$. & Nyctaginiaceae & $\mathrm{H}$ & Pattaraschu & $\mathrm{M} / \mathrm{O}$ \\
\hline 235. & Mitracarpus villosus (Sw.) DC. & Rubiaceae & $\mathrm{H}$ & - & M \\
\hline 236. & Mollugo nudicaulis Lam. & Aizoaceae & $\mathrm{H}$ & Kuthiraipoondu & M \\
\hline 237. & Momordica charantia $\mathrm{L}$. & Cucurbitaceae & $\mathrm{Cl}$ & Pavakaai & M \\
\hline 238. & Morinda coreia Buch.-Ham & Rubiaceae & $\mathrm{T}$ & Manjanati & M \\
\hline 239. & Moringa concanensis Nimmo ex Gibs. & Moringaceae & $\mathrm{T}$ & Kattumurungai & M \\
\hline 240. & Moringa oleifera Lam. & Moringaceae & $\mathrm{T}$ & Murungai & $\mathrm{M} / \mathrm{E}$ \\
\hline 241. & Mucuna pruriens (L.) DC. & Fabaceae & $\mathrm{Cl}$ & Poonaikaali & M \\
\hline 242. & Mukia maderaspatana (L.) M. Roem. & Cucurbitaceae & $\mathrm{Cl}$ & Musumusukkai & M \\
\hline 243. & Mundulea sericea (Willd.) A. Cheval & Fabaceae & $\mathrm{T}$ & Pilavaram & M \\
\hline 244. & Muntingia calabura $\mathrm{L}$. & Elaeocarpaceae & $\mathrm{T}$ & Thenpazham & M \\
\hline 245. & Murraya koenigii (L.) Spreng. & Rutaceae & $\mathrm{T}$ & Kariveppilai & M \\
\hline 246. & Musa paradisiaca $\mathrm{L}$. & Musaceae & $\mathrm{H}$ & Vaazhai & $\mathrm{M} / \mathrm{E}$ \\
\hline 247. & Nicandra physalodes (L.) Gaertn. & Solanaceae & $\mathrm{H}$ & Sudakku thakazhi & M \\
\hline 248. & Nothosaerva brachiata (L.) Wight & Amaranthaceae & $\mathrm{H}$ & Sirupeelai chakkalathi & M \\
\hline 249. & Nyctanthes arbor-tristis $\mathrm{L}$. & Nyctanthaceae & $\mathrm{T}$ & Parijaatham & $\mathrm{M} / \mathrm{O}$ \\
\hline 250. & Ocimum americanum $\mathrm{L}$. & Lamiaceae & $\mathrm{H}$ & Nai thulasi & $\mathrm{M}$ \\
\hline 251. & Ocimum basilicum $\mathrm{L}$. & Lamiaceae & $\mathrm{H}$ & Thiruneetrupatchai & M \\
\hline 252. & Ocimum gratissimum $\mathrm{L}$. & Lamiaceae & $\mathrm{H}$ & Elumicha tulasi & $\mathrm{M}$ \\
\hline 253. & Ocimum tenuiflorum $\mathrm{L}$. & Lamiaceae & $\mathrm{H}$ & Thulasi & M \\
\hline 254. & Oldenlandia umbellata $\mathrm{L}$. & Rubiaceae & $\mathrm{H}$ & Impural & M \\
\hline 255. & Opuntia stricta (Haw.) Haw. & Cactaceae & $\mathrm{H}$ & Chappathikkalli & M \\
\hline 256. & Opuntia vulgaris Mill. & Cactaceae & $\mathrm{H}$ & Chappathikkalli & M \\
\hline 257. & Ottelia alismoides (L.) Pers. & Hydrocharitaceae & $\mathrm{H}$ & Neerkuliri & M \\
\hline 258. & Oxalis corniculata $\mathrm{L}$. & Oxalidaceae & $\mathrm{H}$ & Puliyarai & M \\
\hline 259. & Parkinsonia aculeata $\mathrm{L}$. & Caesalpiniaceae & $\mathrm{T}$ & Parankivelamaram & M \\
\hline 260. & Parthenium hysterophorus L. & Asteraceae & $\mathrm{H}$ & Vishapullu & $\mathrm{M} / \mathrm{Mis}$ \\
\hline 261. & Passiflora foetida $\mathrm{L}$. & Passifloraceae & $\mathrm{Cl}$ & Siruppunaikali & M \\
\hline 262. & $\begin{array}{l}\text { Pavonia procumbens (Wall ex. Wight } \& \\
\text { Arn.) Walp. }\end{array}$ & Malvaceae & $S$ & - & M \\
\hline 263. & Pavonia zeylanica (L.) Cav. & Malvaceae & $\mathrm{H}$ & Sittramuttti & M \\
\hline 264. & Pedalium murex $\mathrm{L}$. & Pedaliaceae & $\mathrm{H}$ & Yanai nerunji & M \\
\hline 265. & Pedilanthus tithymaloides (L.) Poir. & Euphorbiaceae & $\mathrm{H}$ & Kannaadikkallli & M \\
\hline 266. & Peltophorum pterocarpum (DC.) & Caesalpiniaceae & $\mathrm{T}$ & Avalvagai & M \\
\hline 267. & Pentanema indicum (L.) Ling & Asteraceae & $\mathrm{H}$ & Mookutthipoondu & M \\
\hline
\end{tabular}




\begin{tabular}{|c|c|c|c|c|c|}
\hline 268. & Pergularia daemia (Forrsk.) Chiov. & Asclepiadaceae & $\mathrm{Cl}$ & Vaelipparuthi & M \\
\hline 269. & $\begin{array}{l}\text { Peristrophe paniculata (Forssk.) } \\
\text { Brummitt }\end{array}$ & Acanthaceae & $\mathrm{H}$ & Naganantha & M \\
\hline 270. & Phyla nodiflora (L.) Greene & Verbenaceae & $\mathrm{H}$ & Poduthalai & M \\
\hline 271. & Phyllanthus amarus Schum. \& Thonn. & Euphorbiaceae & $\mathrm{H}$ & Kizha-nelli & M \\
\hline 272. & Phyllanthus emblica $\mathrm{L}$. & Euphorbiaceae & $\mathrm{T}$ & Nelli & $\mathrm{M} / \mathrm{E}$ \\
\hline 273. & Phyllanthus maderaspatensis L. & Euphorbiaceae & $\mathrm{H}$ & Nella nelli & M \\
\hline 274. & Phyllanthus reticulatus Poir. & Euphorbiaceae & $S$ & Kanimpoolanthi & $\mathrm{M} / \mathrm{E}$ \\
\hline 275. & Physalis minima $\mathrm{L}$. & Solanaceae & $\mathrm{H}$ & Sodakku thakali & M \\
\hline 276. & Pistia stratiotes $\mathrm{L}$. & Araceae & $\mathrm{H}$ & Agasa-thamarai & M \\
\hline 277. & Pithecellobium dulce (Roxb.) Benth. & Mimosaceae & $\mathrm{T}$ & Kodukkai puli & $\mathrm{M} / \mathrm{E} / \mathrm{Ti}$ \\
\hline 278. & $\begin{array}{l}\text { Plectranthus amboinicus (Lour.) } \\
\text { Spreng. }\end{array}$ & Lamiaceae & $\mathrm{H}$ & Karppuravalli & M \\
\hline 279. & Plumbago auriculata Lam. & Plumbaginaceae & $S$ & Neelakkodi vaeli & M \\
\hline 280. & Plumbago zeylanica $\mathrm{L}$. & Plumbaginaceae & $\mathrm{H}$ & Venkodiveli & M \\
\hline 281. & Polyalthia longifolia (Sonner.) Thw. & Annonaceae & $\mathrm{T}$ & Nettilingam & M \\
\hline 282. & Polycarpaea corymbosa (L.) Lam. & Caryophyllaceae & $\mathrm{H}$ & Nilaisedachi & M \\
\hline 283. & Polygonum glabrum Willd. & Polygonaceae & $\mathrm{H}$ & Aattralari & M \\
\hline 284. & Pongamia pinnata (L.) Pierre & Fabaceae & $\mathrm{T}$ & Punga maram & $\mathrm{M} / \mathrm{Ti}$ \\
\hline 285. & Portulaca oleracea L. & Portulacaceae & $\mathrm{H}$ & Parupu keerai & M \\
\hline 286. & Portulaca pilosa $\mathrm{L}$. & Portulacaceae & $\mathrm{H}$ & Mukkilikeerai & M \\
\hline 287. & Portulaca quadrifida $\mathrm{L}$. & Portulacaceae & $\mathrm{H}$ & Sinnaparupukeerai & M \\
\hline 288. & Pouzolzia zeylanica (L.) Benn. & Urticaceae & $\mathrm{H}$ & Kallurki & M \\
\hline 289. & Priva cordifolia (L.f.) Druce & Verbenaceae & $\mathrm{H}$ & Aadai otti & M \\
\hline 290. & Prosopis juliflora (Sw.) Dc. & Mimosaceae & $\mathrm{T}$ & Vaelikaruvai & $\mathrm{M} / \mathrm{Ti} / \mathrm{Mis}$ \\
\hline 291. & Psidium guajava $\mathrm{L}$. & Myrtaceae & $\mathrm{T}$ & Koyya & $\mathrm{E} / \mathrm{Ti}$ \\
\hline 292. & $\begin{array}{l}\text { Pterolobium hexapetalum (Roth.) Sant. } \\
\& \text { Wagh }\end{array}$ & Caesalpiniaceae & $S$ & Karuendu & $\mathrm{M}$ \\
\hline 293. & Punica granatum $\mathrm{L}$. & Punicaceae & $S$ & Madhulai & $\mathrm{M} / \mathrm{E}$ \\
\hline 294. & Pupalia lappacea (L.) Juss. & Amaranthaceae & $\mathrm{H}$ & Adai-otti & M \\
\hline 295. & Randia dumetorum (Retz) poiret & Rubiaceae & $S$ & Marukkarai & M \\
\hline 296. & Rhynchosia minima (L.) DC. & Fabaceae & $\mathrm{Cl}$ & Kaliyathuvarai & M \\
\hline 297. & Ricinus communis $\mathrm{L}$. & Euphorbiaceae & $S$ & Amanakku & M \\
\hline 298. & Rivea hypocrateriformis (Desr.) Choisy & Convolvulaceae & $\mathrm{Cl}$ & Boodhikeerai & M \\
\hline 299. & Rothia indica (L.) Druce & Fabaceae & $\mathrm{H}$ & Nurreypitten keerai & M \\
\hline 300. & Rungia repens $(\mathrm{L}$.$) Nees$ & Acanthaceae & $\mathrm{H}$ & Kodakasalai & M \\
\hline 301. & $\begin{array}{l}\text { Sansevieria roxburghiana Schultes \& } \\
\text { Schultes }\end{array}$ & Dracaenaceae & $\mathrm{H}$ & Marul & M \\
\hline 302. & Santalum album $\mathrm{L}$. & Santalaceae & $\mathrm{T}$ & Sandanam & $\mathrm{M} / \mathrm{Ti}$ \\
\hline 303. & $\begin{array}{l}\text { Sarcostemma brunonianum Wight \& } \\
\text { Arn. }\end{array}$ & Asclepiadaceae & $\mathrm{Cl}$ & Perum Aattlaankodi & M \\
\hline 304. & Scoparia dulcis $\mathrm{L}$. & Scrophulariaceae & $\mathrm{H}$ & Sarakkotthini & M \\
\hline 305. & Sesamum alatum Thonn. & Pedaliaceae & $\mathrm{H}$ & - & M \\
\hline 306. & Sesbania grandiflora (L.) Poir. & Fabaceae & $\mathrm{T}$ & Agathi & $\mathrm{M} / \mathrm{E} / \mathrm{Ti}$ \\
\hline 307. & Sesbania sesban (L.) Merr. & Fabaceae & $\mathrm{S}$ & Chittagathi & M \\
\hline
\end{tabular}




\begin{tabular}{|c|c|c|c|c|c|}
\hline 308. & Sida acuta Burm.f. & Malvaceae & $\mathrm{H}$ & Arival manai poondu & M \\
\hline 309. & Sida cordata (Burm. f.) Borssum & Malvaceae & $\mathrm{H}$ & Pazhampaasi & M \\
\hline 310. & Sida cordifolia $\mathrm{L}$ & Malvaceae & $\mathrm{S}$ & Nilatutthi & M \\
\hline 311. & Solanum elaeagnifolium Cav. & Solanaceae & $\mathrm{H}$ & - & M \\
\hline 312. & Solanum insanum $\mathrm{L}$. & Solanaceae & $\mathrm{H}$ & Mullu Kathiri & M \\
\hline 313. & Solanum lycopersicum $\mathrm{L}$. & Solanaceae & $\mathrm{H}$ & Thakkali & $\mathrm{E}$ \\
\hline 314. & Solanum nigrum $\mathrm{L}$. & Solanaceae & $\mathrm{H}$ & Milaguthakkali & $\mathrm{M} / \mathrm{E}$ \\
\hline 315. & Solanum pubescens Willd. & Solanaceae & $\mathrm{S}$ & Rameshwarasundai & M \\
\hline 316. & Solanum seaforthianum Andr. & Solanaceae & S & - & M \\
\hline 317. & Solanum surattense Burm.f. & Solanaceae & S & Kandankathiri & M \\
\hline 318. & Solanum torvum $\mathrm{Sw}$. & Solanaceae & $\mathrm{S}$ & Chundai & $\mathrm{M} / \mathrm{E}$ \\
\hline 319. & Solanum trilobatum $\mathrm{L}$. & Solanaceae & $\mathrm{Cl}$ & Thoodhuvalai & M \\
\hline 320. & Sonchus oleraceus L. & Asteraceae & $\mathrm{H}$ & Shaadevi & M \\
\hline 321. & Sopubia delphiniifolia (L.) G.Don & Scrophulariaceae & $\mathrm{H}$ & - & M \\
\hline 322. & Spathodea campanulata Beauv. & Bignoniaceae & $\mathrm{T}$ & Ruthrapalasu maram & $\mathrm{M} / \mathrm{O}$ \\
\hline 323. & Spermacoce hispida $\mathrm{L}$. & Rubiaceae & $\mathrm{H}$ & Nathaichuri & M \\
\hline 324. & Sphaeranthus amaranthoides Burm.f. & Asteraceae & $\mathrm{H}$ & Siva karandhai & M \\
\hline 325. & Sphaeranthus indicus $\mathrm{L}$. & Asteraceae & $\mathrm{H}$ & Kotta karandhai & M \\
\hline 326. & Spilanthes calva DC. & Asteraceae & $\mathrm{H}$ & Palvalipoondu & M \\
\hline 327. & Stachytarpheta jamaicensis (L.) Vahl & Verbenaceae & $\mathrm{H}$ & Seemai nayuruvi & M \\
\hline 328. & Striga asiatica (L.) Kuntze & Scrophulariaceae & $\mathrm{H}$ & Pulluruvi & M \\
\hline 329. & $\begin{array}{l}\text { Strobilanthes consanguineaus (Nees) } \mathrm{T} \text {. } \\
\text { Anders. }\end{array}$ & Acanthaceae & $\mathrm{H}$ & Perunkurinji & M \\
\hline 330. & Strychnos nux-vomica $\mathrm{L}$. & Loganiaceae & $\mathrm{T}$ & Yetti & M \\
\hline 331. & Strychnos potatorum $\mathrm{L}$. & Loganiaceae & $\mathrm{T}$ & Thaetraan kottai & M \\
\hline 332. & Swietenia macrophylla King & Meliaceae & $\mathrm{T}$ & Mahagony & $\mathrm{M} / \mathrm{Ti}$ \\
\hline 333. & Synadenium grantii Hook.f. & Euphorbiaceae & $\mathrm{S}$ & - & M \\
\hline 334. & Synedrella nodiflora (L.) Gaertn. & Asteraceae & $\mathrm{H}$ & Mudiyarthirapachai & M \\
\hline 335. & Syzygium cumini (L.) Skeels & Myrtaceae & $\mathrm{T}$ & Navaal & $\mathrm{M} / \mathrm{E}$ \\
\hline 336. & Tabebuia rosea (Bertol.) DC. & Bignoniaceae & $\mathrm{T}$ & Nagasenbagamaram & $\mathrm{O}$ \\
\hline 337. & Tamarindus indica $\mathrm{L}$. & Caesalpiniaceae & $\mathrm{T}$ & Puliya maram & $\mathrm{M} / \mathrm{E} / \mathrm{Ti}$ \\
\hline 338. & Tecoma stans $(\mathrm{L}$.$) Kunth$ & Bignoniaceae & $\mathrm{T}$ & Sornapatti & M \\
\hline 339. & Tectona grandis L.f. & Verbenaceae & $\mathrm{T}$ & Thekku & $\mathrm{T}$ \\
\hline 340. & Tephrosia purpurea (L.) Pers. & Fabaceae & $\mathrm{H}$ & Kozhinji & M \\
\hline 341. & $\begin{array}{l}\text { Terminalia arjuna } \\
\text { (Roxb.) Wight \& Arn. }\end{array}$ & Combretaceae & $\mathrm{T}$ & Marutha maram & M \\
\hline 342. & Terminalia catappa $\mathrm{L}$. & Combretaceae & $\mathrm{T}$ & Naattu Badaam & M \\
\hline 343. & Terminalia cuneata Roxb. & Combretaceae & $\mathrm{T}$ & Neer Marudhu & - \\
\hline 344. & $\begin{array}{l}\text { Thespesia populnea (L.) Soland ex } \\
\text { Correa }\end{array}$ & Malvaceae & $\mathrm{T}$ & Poovarasu & M \\
\hline 345. & Thevetia peruviana K.Schum & Apocynaceae & $\mathrm{T}$ & Ponnarali & M \\
\hline 346. & $\begin{array}{l}\text { Tinospora cordifolia (Willd.) Miers ex } \\
\text { Hook. f. \& Thoms. }\end{array}$ & Menispermaceae & $\mathrm{Cl}$ & Seendhil & M \\
\hline 347. & Tithonia diversifolia (Hemsl.) A. Gray & Asteraceae & $\mathrm{S}$ & Valsuriyagandhi & $\mathrm{M}$ \\
\hline
\end{tabular}




\begin{tabular}{|c|c|c|c|c|c|}
\hline 348. & Trianthema decandra $\mathrm{L}$. & Aizoaceae & $\mathrm{H}$ & Vellai saranai & $\mathrm{M} / \mathrm{E}$ \\
\hline 349. & Trianthema portulacastrum $\mathrm{L}$. & Aizoaceae & $\mathrm{H}$ & Saranai & $\mathrm{M} / \mathrm{E}$ \\
\hline 350. & $\begin{array}{l}\text { Tribulus subramanii P. Singh, Giri \& V. } \\
\text { Singh }\end{array}$ & Zygophyllaceae & $\mathrm{H}$ & Periyanerunji & M \\
\hline 351. & Tribulus terrestris $\mathrm{L}$. & Zygophyllaceae & $\mathrm{H}$ & Nerinjimul & M \\
\hline 352. & Trichodesma indicum (L.) R. Br. & Boraginaceae & $\mathrm{H}$ & Kavil thumbai & M \\
\hline 353. & $\begin{array}{l}\text { Trichodesma zeylanicum (Burm.f.) R. } \\
\text { Br. }\end{array}$ & Boraginaceae & $\mathrm{H}$ & Kaluthai thumbai & M \\
\hline 354. & Tridax procumbens $\mathrm{L}$. & Asteraceae & $\mathrm{H}$ & Vettukayapoodu & M \\
\hline 355. & Typha angustifolia $\mathrm{L}$. & Typhaceae & $\mathrm{H}$ & Sambu & M \\
\hline 356. & Urena lobata $\mathrm{L}$. & Malvaceae & $S$ & Ottatthi & M \\
\hline 357. & Vernonia anthelmintica (L.) Willd. & Asteraceae & $\mathrm{H}$ & Kattu-seeragam & M \\
\hline 358. & Vernonia cinerea (L.) Less. & Asteraceae & $\mathrm{H}$ & Mookutthipoondu & M \\
\hline 359. & Vigna trilobata (L.) Verdc. & Fabaceae & $\mathrm{H}$ & Panipayir & M \\
\hline 360. & Vitex negundo L. & Verbenaceae & $\mathrm{T}$ & Nochi & M \\
\hline 361. & Waltheria indica $\mathrm{L}$. & Sterculiaceae & $\mathrm{H}$ & Chembudu & M \\
\hline 362. & Wattakaka volubilis (L.f.) Stapf & Asclepiadaceae & $\mathrm{Cl}$ & Kodipaalai & M \\
\hline 363. & Wedelia chinensis (Osbeck) Merr. & Asteraceae & $\mathrm{H}$ & Manjal Karisalankanni & M \\
\hline 364. & Withania somnifera (L.) Dunal & Solanaceae & $\mathrm{H}$ & Amukkira & M \\
\hline 365. & Wrightia tinctoria (Roxb.) R.Br & Apocynaceae & $\mathrm{T}$ & Vetpaalai & M \\
\hline 366. & Xanthium indicum Koen. & Asteraceae & $\mathrm{H}$ & Marul oomatham & M \\
\hline 367. & Ziziphus mauritiana Lam. & Rhamnaceae & $\mathrm{T}$ & Illandhai & $\mathrm{M} / \mathrm{E}$ \\
\hline 368. & Ziziphus oenoplia (L.) Mill. & Rhamnaceae & $\mathrm{T}$ & Soorai pazham & $\mathrm{M} / \mathrm{E}$ \\
\hline 369. & Ziziphus xylopyrus (Retz.) Willd. & Rhamnaceae & $\mathrm{T}$ & Kottailandhai & M \\
\hline 370. & Zornia gibbosa Span. & Fabaceae & $\mathrm{H}$ & Arundhalai otti & M \\
\hline
\end{tabular}

H- Herb; S-Shrub; Cl-Climber; T-Tree; M-Medicine; E-Edible; O-Ornamental; Ti-Timber; Mis-Miscellaneous.

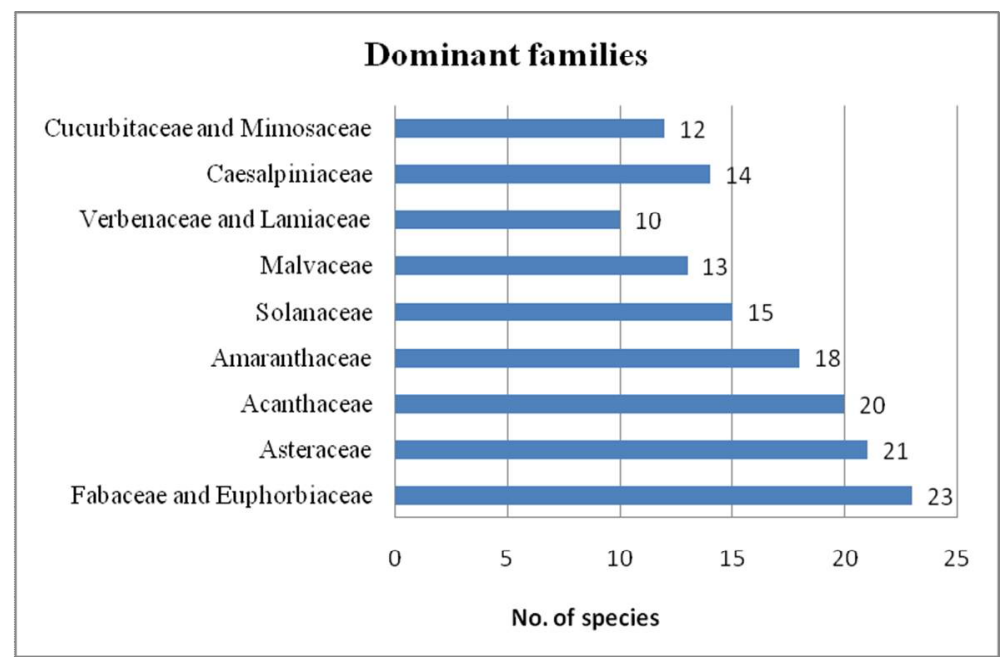

Fig. 1. Dominant families were recorded from the study area. 

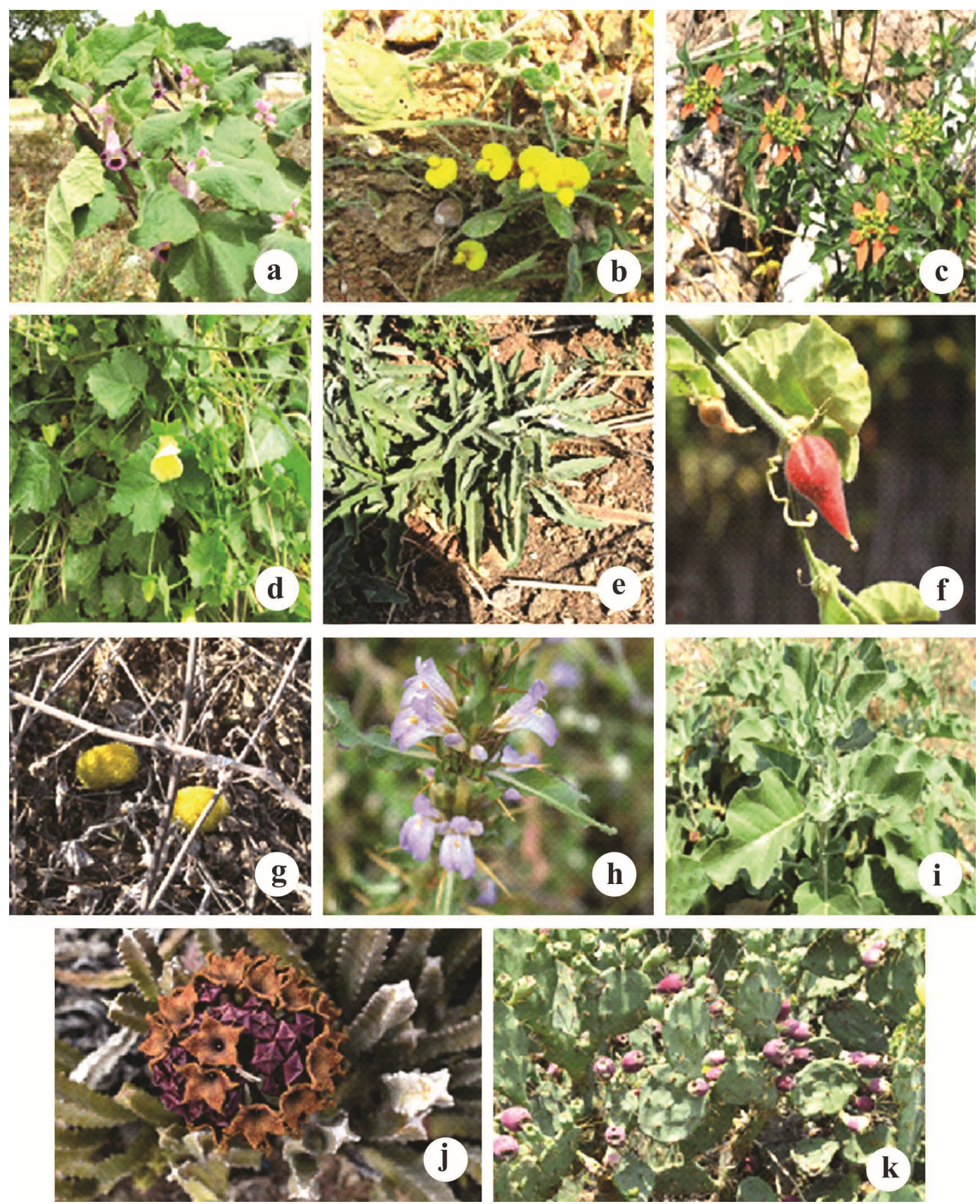

Fig. 2 a-k: Some common taxa recorded in phenological stage: a) Martynia annua L. b) Crotalaria globosa Wight \& Arn. c) Euphorbia cyathophora Murr. d) Pavonia procumbens (Wall ex. Wight \& Arn.) Walp e) Solanum elaeagnifolium Cav. f) Kedrostis foetidissima (Jacq.) Cogn. g) Cucumis dipsaceus Ehrenb. ex Spach. h) Hygrophila schulli (Hamilt.) M.R.Almeida \& S.M. Almeida i) Withania somnifera (L.) Dunal j) Caralluma umbellata Haw. k) Opuntia vulgaris Mill. 

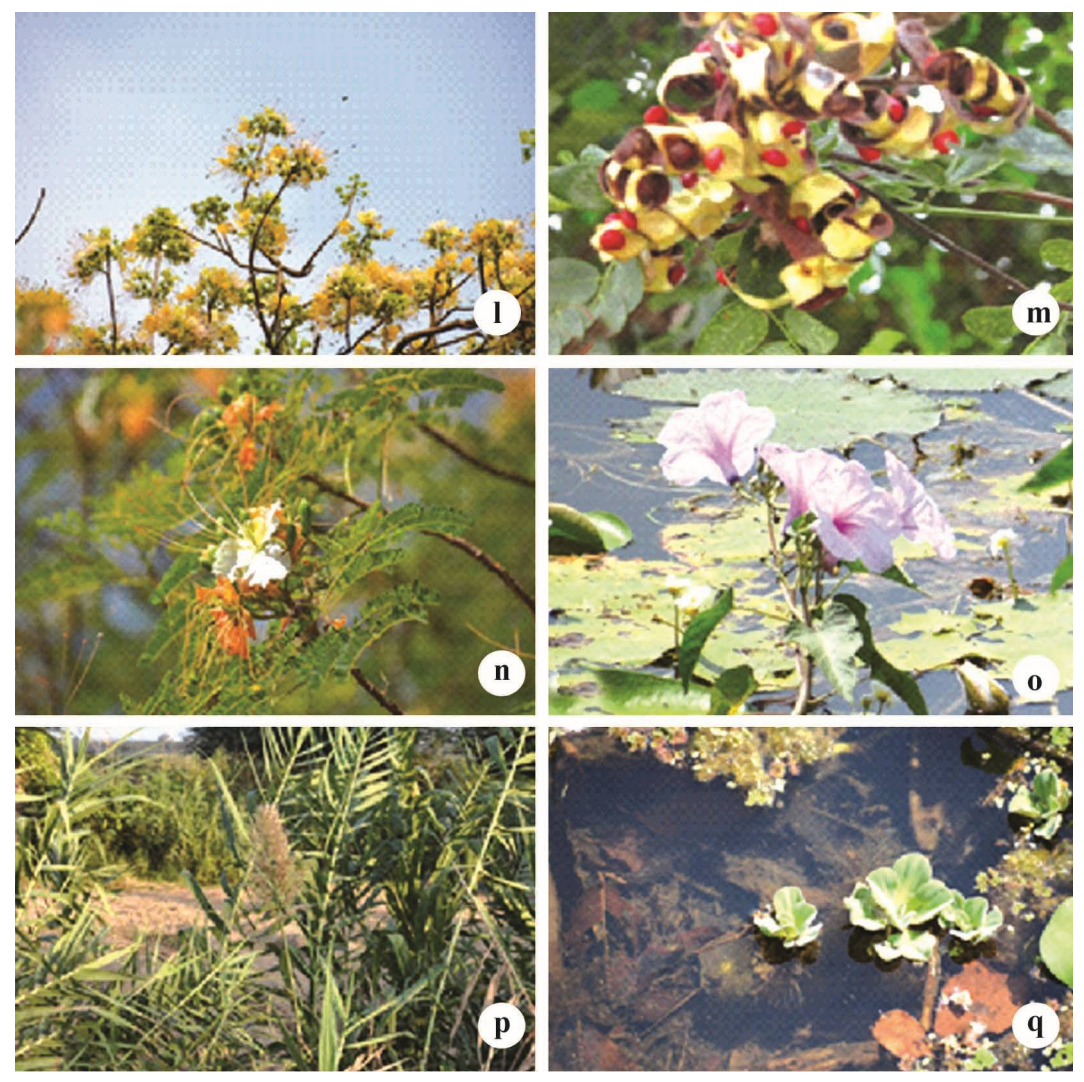

Fig. 3 1-q: Some common taxa recorded in phenological stage: 1) Crateva adansonii DC. m) Adenanthera pavonina L. n) Delonix elata (L.) Gamble o) Ipomoea carnea Jacq. p) Arundo donax L. q) Pistia stratiodes $\mathrm{L}$.

Urbanisation affects the natural flora and fauna that imposes to document the existing biodiversity that enable to conserve them from the extinction (Dolan et al., 2011). The flora and ecosystem of this region are facing serious threats due to the increased anthropogenic activities. Natural flora of Udumalpet taluk of Tiruppur district are facing serious threats especially because of the growing attention of its tourist places, such as Tirumoorthy hills, Amaravathy dam, Anamalai tiger reserve etc., and due to the constructions of new inns, hotels, and extension of roads etc. It has been noted that, a number of timber yielding and medicinally important trees such as Neem (Azadirachta indica A. Juss.), Tamarind (Tamarindus indica L.), Gulmohar (Delonix regia (Boj. ex Hook) Rafin.) are uprooted for road construction and highway extension programmes. Frequent documentation are done on the flora of the protected areas (Shankaranarayanan and Gupta, 1959; Vajravelu and Joesph, 1971; Balasubramanian et al., 1997; Fischer, 2004; Rasingam, 2012; Murugeshwaren et al., 2014; Ramachandran et al., 2014; Sarvalingam and Rajendran, 2018), but little attention is given to the flora of rural and aboriginal areas that are under different threats. Urbanization, habitat fragmentation, anthropological pressures, and pollution are the profound reasons for the destruction of the natural vegetation in the rural and aboriginal areas which is considered as an important component of the healthy environment. 
In Tiruppur District, Udumalpet Taluk is the main source of electricity generated through wind mills and wind turbines. The occurrence of wind mills, affect the natural vegetation as well as cultivated fields. Thus, documentation of the existing flora of Udumalpet taluk will be helpful for conservation of its important natural plant resources.

\section{Acknowledgements}

The authors are thankful to the Director General, Central Council for Research in Siddha (Ministry of AYUSH, Govt. of India) Chennai for the constant encouragement and thankful to CCRS for sanctioning and granting the IMR Project. We also acknowledge the In-charge of SCRU, Palayamkottai for her constant encouragement and support. We thank to Mr. C.Maharaja and K.Karthik Field Assistants for their service during field surveys.

\section{References}

Anuradha, G. and Muruganandam, A. 2016. Floristic Inventory on Nagamangalam Ayyanaar Sacred Grove at Ariyalur District, Tamil Nadu. Int. J. Modn. Res. Revs. 4(6): 1172-1176.

Balasubramanian, P., Rajasekaran, A. and Prasad, S.N. 1997. Folk medicine of the Irulas of Coimbatore forests. Ancient Sci. Life 16(3): 222-226.

Chandrabose, M. and Nair, N.C. 1987. Flora of Coimbatore. Bishen Singh Mahendra Pal Singh, Dehra Dun.

Dolan, R.W., Moore, M.E. and Stephens, J.D. 2011. Documenting effects of urbanization on flora using herbarium records. J. Ecol. 99(4): 1055-1062.

Fischer, C.E.C. 2004. The Flora of Anaimalai hills Coimbatore District, Madras. Bishen Singh Mahendra Pal Singh, Dehra Dun.

FRLHT . http://envis.frlht.org/bot. (envis.frlht.org / frlhtenvis.nic.in). FRLHT's ENVIS Centre on Medicinal Plants, Bengaluru. (Accessed on 27 December 2018)

Gamble, J.S. and Fischer, C.E.C. 1915-1936. The Flora of Presidency of Madras. Vols. 1-3. Adlard and Son Ltd, London.

Ganorkar, R.P. and Kshirsagar, A.D. 2013. Floristic Study of Shirur Region Pune, Maharashtra. Int. Res. J. Biol. Sci. 2(5): 78-82.

George, P., Arekar, C. and Subashini, D. 2011. Biodiversity survey of trees and ornamental plants in Karunya University, Coimbatore, India. Int. J. Biodiver. Conserv. 39: 431-433.

Gurusamy, S, Sarvalingam, A. and Rajendran, A. 2016. Vascular Floristic Composition of Sadhuragiri Hills in the Southern Western Ghats of Tamil Nadu, India. Int. J. Adv. Res. Biol. Sci. 3(1): 149-160.

Henry, A.N., Kumari, G.R. and Chithra V. 1987. Flora of Tamil Nadu, India. Series 1: Analysis. Vol. 2. Botanical Survey of India, Coimbatore.

Henry, A.N., Chithra, V. and Balakrishnan, N.P. 1989. Flora of Tamil Nadu, India, Series-I, Analysis. Vol. 3. Botanical Survey of India, Southern Circle, Coimbatore.

IUCN, 2018. The IUCN Red List of Threatened Species 1998: e. T31852A9665066. http://dx.doi.org/ 10.2305/IUCN.UK.1998.RLTS.T31852A9665066.en. Downloaded on 17 December 2018.

Jain, S.K. and Rao, R.R. 1977. A handbook of field and herbarium methods. Today and Tomorrow's Printers and Publishers, New Delhi, India.

Kumar, D. S. and Ritesh, S. 2014. Study of angiospermic wall floristic composition of city Buxar, (Bihar) India. J. Pharmacog. Phytochem. 2(5): 52-54.

Mantosh K.S. 2013. A Floristic Study of Koria District (Chhattisgarh) India. Int. J. Scientific Res. Pub. 3(4):1-5.

Matthew, K.M. 1981-1983. Flora of Tamil Nadu Carnatic. The Rapinat Herbarium, St. Joseph's College, Tiruchirapalli, Tamil Nadu. India.

Matthew, K.M. 1991. An Excursion flora of Central Tamil Nadu, India.Oxford and IBH Publishing Co., New Delhi. 
Murugeshwaren, R., Rajendren, A., Venkadesan, K. and Hafiz, C.M.A. 2014. Diversity of Unani medicinal plants in Southern Western Ghats of Coimbatore District, Tamil Nadu, India. Int. J. Herbal Med. 2(1): $29-38$.

Nair N.C. and Henry, A.N. 1983. Flora of Tamil Nadu, India. Series I: Analysis. Vol. 1. Botanical Survey of India, Coimbatore.

Parthipan, B., Rajeeswari, M. and Jeeva, S. 2016. Floristic Diversity of South Travancore Hindu College (S. T. Hindu College) Campus, Kanyakumari District (Tamil Nadu) India. Biosci. Discovery. 7(1): 41-56.

Rajendran, A., Aravindhan, V. and Sarvalingam, A. 2014. Biodiversity of the Bharathiyar University campus, India: A floristic approach. Int. J. Biodiver. Conserv. 6(4): 308-319.

Ramachandran, V.S., Selvalakshmi, S. and Betty, T. 2014. Floral diversity of Karian Shola MPCA, Coimbatore District, Tamil Nadu, with special emphasis on the conservation of RET and endemic plants of Anamalai hills. Elixir. Appl. Botany 66: 20653-20655.

Ramachandran, V.S. 2007. Wild edible plants of the Anamalais, Coimbatore district, Western Ghats, Tamil Nadu. Ind. J. Trad. Knowl. 6(1): 173-176.

Rasingam, L. 2012. Ethnobotanical studies on the wild edible plants of Irula tribes of Pillur Valley, Coimbatore District, Tamil Nadu, India. Asian Pacific J. Trop. Biomed. S1493-S1497.

Sandifer, P.A., Sutton-Grier, A.E. and Ward, B.P. 2015. Exploring connections among nature, biodiversity, ecosystem services, and human health and well-being: Opportunities to enhance health and biodiversity conservation. Ecosys. Serv.12: 1-15.

Sarvalingam, A. and Rajendran, A. 2018. Climbing plants of the Southern Western Ghats of Coimbatore in India and their Economic uses. American - Eurasian Journal of Agric and Environ. Sci. 15(7): 13121322.

Sasidharan, N. 2004. Biodiversity Documentation for Kerala. Part 6: Flowering Plants. Kerala Forest Research Institute, Peechi.

Savita S.R. and Sanjaykumar, R.R.2017. Floristic diversity of Bhimashankar Wildlife Sanctuary, northern Western Ghats, Maharashtra, India. J. Threatened Taxa. 9(8): 10493-10527.

Shankaranarayanan, K.A. and Gupta R.K. 1959. The vegetation of Coimbatore District. Indian Forester. 85(9): 533-541.

Singh, K.K.1982. Floristic elements in the vegetation of Kheri District Uttar Pradesh, India. J. Econ. Taxon. Bot. 3(2): 557-564

Sinha, B.K. 2005 Floristic diversity and vegetation composition of Lakh-Bahosi wetlands of Kannauj, Uttar Pradesh. Phytotax. 5: 106-115.

Sridharan, A. and Kalpana, S.2017. Socio cultural and behavioral Approach of Tribals of Anamalai Hills in Tamil Nadu - a Bicultural Skill Approach. J. Humanities Soc. Sci. 22(11): 74-78.

Sukumaran, S., Jeeva, S., Sobhana Raj, A.D. and Kannan, D. 2008. Floristic Diversity, Conservation Status and Economic Value of Miniature Sacred Groves in Kanyakumari District, Tamil Nadu, Southern Peninsular India. Turk. J. Bot. 32: 185-199.

The Plant List, 2013. Version 1.1. The Plant List, a working list of all plant species. Version 1.1. (Accessed on 27 December 2018)

Vajravelu, E. and Joesph, J. 1971. Addition to the flora of Anamalai Hills, Coimbatore District, Tamil Nadu. Nelumbo. 13(3-4): 264-273.

Vijay, V.W.and Ashok, K.J. 2013. Floristic Diversity of Jhabua District, Madhya Pradesh, India. Acad. J. Plant Sci. 6(4): 146-167. 